\title{
Peuples mobiles : configurations transversales
}

\section{Engin Isin}

\section{(2) OpenEdition}

\section{Journals}

Édition électronique

URL : http://journals.openedition.org/anthropodev/856

DOI : 10.4000/anthropodev.856

ISSN : 2553-1719

\section{Éditeur}

Presses universitaires de Louvain

\section{Édition imprimée}

Date de publication : 1 décembre 2019

Pagination : 129-144

ISBN : 978-2-87558-940-8

ISSN : 2276-2019

\section{Référence électronique}

Engin Isin, «Peuples mobiles : configurations transversales », Anthropologie \& développement [En ligne], 50 | 2019, mis en ligne le 19 décembre 2020, consulté le 25 janvier 2021. URL : http:// journals.openedition.org/anthropodev/856 ; DOI : https://doi.org/10.4000/anthropodev.856

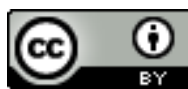

La revue Anthropologie \& développement est mise à disposition selon les termes de la Licence Creative Commons Attribution 4.0 International. 


\title{
Peuples mobiles : configurations transversales
}

\author{
Engin Isin ${ }^{1}$
}

Cet essai tente de penser les « peuples mobiles » comme concept politique. Je considère les peuples mobiles comme norme et non exception, davantage comme des sujets politiques que des peuples sujets. Après avoir discuté la tension entre «peuples » et "mobiles", je m'appuierai sur l'ontologie historique de Ian Hacking pour comprendre comment un peuple advient. Et pour comprendre l'advenir $d u$ peuple, ou plutôt la tension entre un peuple qui se constitue comme un tout et ces peuples qui restent à la marge, je m'appuie sur Giorgio Agamben, Jacques Rancière et Ernesto Laclau, qui ont identifié cette tension comme problème fondamental de la pensée politique " occidentale ». Toutefois, leur indifférence au territoire me rapproche des travaux de James Scott sur les premiers États, qui remettent en question notre appréhension usuelle des peuples comme initialement sédentaires. Sa description du mécanisme selon lequel les "barbares" (peuples mobiles) finissent par être considérés comme une menace envers les peuples sédentaires nous permet de comprendre cette tension. Dès lors s'ouvre la voie à une réflexion sur les peuples mobiles en tant que concept politique.

This essay is an attempt to think "mobile peoples" as a political concept. I consider mobile peoples as a norm rather than an exception and as political subjects rather than subject peoples. After discussing the tension between "mobile" and "peoples", I draw on Ian Hacking's historical ontology for understanding how a people comes to be. For understanding how the people comes to be, or rather, how the tension between a people that constitutes itself as a whole and those peoples that remain as residual parts, I draw on Giorgio Agamben, Jacques Rancière, and Ernesto Laclau as authors who identified this tension as a fundamental problem of "Western" political thought. Yet, their inattention to territory draws me to James Scott whose work on early states challenges how we have come to understand the people as sedentary in the first place. His account of how "barbarians" (mobile peoples) came to be seen as a threat to sedentary peoples enables us to understand that tension. Then a path opens toward thinking about mobile peoples as a political concept.

\footnotetext{
${ }^{1}$ Professeur de politique internationale, Queen Mary University of London (QMUL) et Institut de I'université de Londres à Paris (ULIP) ; engin.isin@qmul.ac.uk
} 


\section{Introduction $^{2}$}

Au cours des deux dernières décennies, les recherches en matière d'études migratoires, réfugiés, citoyenneté et mobilité, ont montré que la mobilité humaine repose sur des mouvements complexes: migrations itératives entre pays, relocalisations de masse et déplacements dans un même pays et entre les villes et régions, géographies mixtes complexes et inégales, histoires imbriquées de mouvements récurrents et saisonniers, usage accru des technologies mobiles dans des pratiques de gouvernance et résistance, recoupement d'identités affectives, culturelles et politiques, formations hybrides culturelles, linguistiques et sociales (Cresswell, 2006 ; Elliott et Urry, 2010 ; Hannam et al., 2016; Merriman, 2012). Ces recherches ont déporté notre attention de la migration décrite comme mouvement à sens unique généralement par-delà et à l'intérieur des frontières étatiques. Cette attention se porte désormais sur le fait que les peuples mobiles, dans leurs multiples facettes et de multiples façons, créent de nouvelles vies sociales, politiques et culturelles, de la connaissance et des pratiques qui apportent un changement politique et deviennent un problème (Adey et al., 2014 ; Gold et Nawyn, 2013 ; Isin et Nyers, 2014).

Ainsi, les universitaires accueillent les chiffres souvent cités de 250 millions de personnes vivant hors de leur pays de naissance (en 2015), soit une augmentation de $41 \%$ depuis 2000, d'un œil relativement sceptique. Car il ne s'agit pas là de simples descriptions de la mobilité humaine (ONU, 2016). Ce sont des désignations qui façonnent notre compréhension de la mobilité comme mouvement à sens unique entre ou au-delà des États. Les utilisations de statistiques pour les politiques publiques de migration et d'immigration, qui concernent généralement la mobilité humaine comprise comme un mouvement à sens unique, sont hérissées de luttes sur le sens et les fonctions de ces chiffres (Boswell, 2009). De plus en plus d'études critiquent le fait que la migration, l'immigration et la mobilité deviennent des problèmes à gérer, résoudre et endiguer, au lieu de penser les peuples mobiles comme sujets politiques créant de nouvelles formes de vie (Geiger, 2013 ; Manderscheid, 2016; Pécoud, 2015). D’autres études portent un regard critique sur le fait que la mobilité s'établirait indépendamment de la participation réfléchie du peuple en tant qu'agent sur l'ensemble du spectre qui va de mobile à immobile (Frello, 2008). C'est peut-être à cause de ce changement que les autorités, agences, et organisations nationales et internationales commencent à admettre que, loin

\footnotetext{
${ }^{2}$ Cet article a été publié en anglais dans le numéro "The Transformative Forces of Migration: Refugees and the Re-Configuration of Migration Societies » édité par Ulrike Hamann et Gökçe Yurdakul (université Humboldt de Berlin, Allemagne). L'article a été traduit par Eugénie Hugo et relu par Magali Chelpi-den Hamer et Éric Komlavi Hahonou, et a servi de support à une conférence donnée lors du colloque 2018 de I'APAD.

$\mathrm{NdT}$ : Le terme people en anglais fait à la fois référence aux termes français " peuple », " population » et "gens ». Nous avons choisi d'utiliser, à quelques exceptions près, le terme "peuple " par souci de cohérence. Les références des ouvrages (numéros de page, années de publication, etc.) sont celles de la version originale en anglais. Les traductions des citations en anglais sont celles du traducteur, sauf mention contraire.
} 
d'être un problème à résoudre ou à gérer, la mobilité des peuples façonne les rapports de la vie politique contemporaine.

Il est encourageant de constater que ces études critiques font évoluer notre point de vue sur les peuples en mouvement de l'eurocentrisme et État-centrisme (Lucassen et Lucassen, 2014, 2017). Il est peut-être temps de poser une question historique, à savoir pourquoi les "peuples mobiles » ont été constitués comme exception (et problème) dès l'origine. Ceci pourrait supposer une réflexion plus large sur les peuples mobiles selon les termes d'Élizabeth Zanoni (2017) qui décrivait alors les travaux précurseurs de Donna Gabaccia (1999). J'affirme ici que des enquêtes généalogiques du concept de " peuple » et des fonctions qu'il en est venu à exercer sont nécessaires. II est certes possible d'approcher cette question par différents biais, mais une approche historique des " peuples mobiles » en tant que concept politique nécessite deux changements.

Le premier, l'exploration des possibilités de reconnaissance des « peuples mobiles » en tant que norme et non exception, va à l'encontre de l'optique dominante. Le concept de " peuple » dénote déjà en soi l'immobilisme, la sédentarisation et un corps politique enfermé dans un territoire délimité. Ainsi, si l'on considère les " peuples mobiles » comme la norme, il nous faut explorer la façon dont le concept " peuple » a acquis initialement sa signification dominante. Le deuxième, l'élaboration de "peuples mobiles " comme concept politique restaurant la subjectivité politique des peuples mobiles, nécessite l'exploration des conditions par lesquelles les " peuples mobiles " sont devenus objets de gouvernement. II faut pour cela reconnaître les difficultés qu'impliquent le concept de " peuple » et les différences entre peuple et peuples en tant que parties.

Le premier changement, considérer les "peuples mobiles " comme la norme et non l'exception, peut sembler plus simple que le second, considérer les "peuples mobiles » comme sujets politiques. Nous pouvons, par exemple, affirmer que les humains se sont toujours déplacés (Feldman, 2015 ; Panayi et Virdee, 2011). Ou, comme l'affirme Jürgen Osterhammel (2009), que l'émergence de mouvements " modernes " de population aux $X I X$ et $X X^{e}$ siècles est le fondement de notre ère. Ou nous pouvons en appeler à la singularité du $X X I^{e}$ siècle et affirmer que le concept de " peuples mobiles " couvre non seulement ceux pour qui traverser toutes sortes de frontières est essentiel à un mode de vie mais également ceux dont les vies sont mêlées aux vies de ceux qui se déplacent. Nous incluons là ceux qui se déplacent de force ou par choix, ceux qui coupent les ponts ou restent en contact, ceux qui reviennent une ou de nombreuses fois, au même titre que ceux qui sont restés sur place. Nous pouvons affirmer qu'il existe différentes catégories de peuples mobiles, notamment les diplomates, familles, investisseurs, retraités, réfugiés, étudiants, voyageurs, touristes, travailleurs qui résident ou endossent ces différentes mobilités. Nous acceptons comme un fait social, par exemple, que les grands-parents dont la subsistance dépend des sommes envoyées par leurs petits-enfants migrants font tout autant partie de cette mobilité que leurs petits-enfants. Nous pouvons affirmer que la mobilité façonne davantage que les vies de ceux qui se déplacent. En conséquence, nous pouvons affirmer qu'elle a également un impact sur les institutions politiques et sociales, ainsi que les pratiques par lesquelles les gens arrangent et gouvernent leur vie. 
Nous pouvons avancer tous ces arguments. Cependant, je me demande si nous n'accordons pas là une trop grande importance à des faits ostensibles au lieu d'interroger les changements de nos points de vue sur les peuples vivant des vies mobiles ? En d'autres termes, n'est-il pas problématique de recourir à des faits autonomes sans prendre en considération les conditions dans lesquelles ces faits se sont produits (Buscher et al., 2011 ; Cresswell, 2006 ; Elliott et Urry, 2010) ? Ne devrions-nous pas nous garder d'y recourir si nous ne demandons ni comment, ni où, ni quand ces peuples mobiles sont devenus une préoccupation voire un problème pour les diverses autorités gouvernementales, ni sous quelles nouvelles modalités ces peuples mobiles sont gouvernés (Endres et al., 2016) ? Si nous devons articuler les effets transformatifs des peuples vivant des vies mobiles à la vie politique du présent, il nous faudra adopter une démarche critique envers les termes dans lesquels nous décrivons les peuples mobiles. Ainsi, plutôt que de recourir pour les peuples mobiles au concept de norme et non d'exception basée sur des faits indépendants et ostensibles, je discuterai de la façon dont nous devrions approcher le concept de "peuple » avant toute chose. Voilà pourquoi les deux prochains paragraphes formeront le gros de cet essai en examinant les généalogies politiques du concept de peuple, d'abord " un peuple » et ensuite "le peuple ». Nous verrons que le glissement $d^{\prime}$ ' " un peuple " vers "le peuple " n'est pas seulement un glissement par lequel un peuple devient souverain politique mais également sujet politique sédentaire (par opposition à mobile).

\section{Qu'est-ce qu' un peuple?}

Qu'est-ce qu'un peuple ? II s'agit d'une question difficile puisque nommer un peuple est toujours un acte plus performatif que descriptif. L'acte de nommer est double : nommer un peuple mobilise en même temps les dénommés à agir en tant que peuple. À ce sujet, l'approche performative de lan Hacking sur la façon dont les types de peuples apparaissent dans la langue et dont cette langue invite les peuples à en devenir un est extrêmement utile. Hacking circonvient ce processus en utilisant deux termes : façonner un peuple ${ }^{3}$ et l'effet de boucle (Hacking, $\left.2007: 289-290\right)$. Ces deux termes sont inspirés de la compréhension performative des relations entre les mots et les choses que Hacking appelle ontologie historique, à la fois pour indiquer sa dette envers Michel Foucault (1997) et pour signifier sa propre démarche, qu'il appelle le "nominalisme dynamique ». L'ontologie historique doit aussi beaucoup à Friedrich Nietzsche (2001) et John Austin (1962), cependant je ferai abstraction de ces influences pour cette discussion et j'analyserai brièvement Hacking, dont les travaux sont essentiels à la question que je traite ici.

Hacking part de l'hypothèse que les mots et les choses sont des effets réciproques (Hacking, 2002 : 3). Pour comprendre ces effets, nous devons comprendre les êtres et

\footnotetext{
${ }^{3} \mathrm{NdT}$ : la traduction de lan Hacking est « façonner les gens ». Nous gardons le terme « peuple » par souci de cohérence. Voir: https://www.college-de-france.fr/media/ian-hacking/UPL6120975782849689510_ Hacking2004_2005.pdf (consulté le 03/11/2019).
} 
l'advenir des êtres comme un parcours historique. Les êtres qui deviennent des choses telles les classifications, idées, peuples, ou institutions sont tous objets de l'ontologie historique dans le sens où nous ne pouvons comprendre ces choses sans en retracer I'histoire. Ces objets n'ont de sens que dans la mesure où nous comprenons comment ils sont advenus et ont acquis leurs significations. Par exemple, Hacking écrit que « l'idée que les peuples se séparent naturellement en grands groupes raciaux, ethniques ou linguistiques est largement le produit d'une invention récente, l'État-nation " (Hacking, 2007 : 289). Rien ne porte à croire que nous trouverons ces choses dans le passé de la même manière que nous les comprenons aujourd'hui, ou que ces choses du présent sont des versions évoluées de choses du passé. Ainsi, l'ontologie historique considère que les "types de peuples » adviennent historiquement en tant que descriptions inventées par lesquelles le peuple se constitue en êtres agissants. De telles descriptions s'ancrent dans les pratiques humaines et apportent de nouvelles façons d'agir et d'être au monde. Tous les actes sont des actes performés au moyen d'une description. Si de nouvelles descriptions apparaissent, de nouvelles possibilités d'action apparaissent à leur tour (Hacking, 2002 : 108). C'est dans ce sens que l'acte de nommer un peuple est un acte de "façonner les gens ". Si le peuple ne reprend pas ces descriptions à son compte et n'agit pas à leur aune en interaction réciproque, la description n'a aucune force performative. Lorsqu'un peuple agit, il interagit avec des descriptions, ce qui crée un effet de boucle (ou de retour) où les descriptions acquièrent une valeur performative. Les noms (mots) et les nommés (choses) interagissent. Hacking insiste sur le fait que ce processus est dynamique; dans l'effet de boucle, il n'y a aucun moment statique où l'on peut dire que le nommé est l'effet du nom ou vice versa. Au lieu de cela, le nom et le nommé se constituent de façon dynamique.

Hacking nomme cinq moments dynamiques par lesquels mots et choses deviennent effets réciproques. Premièrement, une description apparaît sur un type de peuple. Deuxièmement, le peuple commence à agir selon cette description. Troisièmement, des institutions font leur apparition pour prendre en charge les types de peuples agissant selon cette description. Quatrièmement, des connaissances sur le type de peuple et ses caractéristiques apparaissent: féroce ou docile, artistique ou guerrier, artisan ou chasseur, etc. Cinquièmement, les autorités, l'expertise, l'administration du peuple surgissent et règlementent le type de peuple agissant selon cette description. Une ontologie historique d'un type de peuple entend qu'on enquête sur la façon et les moments où ces cinq moments dynamiques ont existé et sur la façon dont cette description a été rendue possible puis transformée. Hacking donne des exemples de différents types de peuples (Hacking, 2007 : 285). Il évoque notamment l'avalanche de chiffres au XIX siècle concernant les catégories de population: assassins, voleurs, prostituées, ivrognes, vagabonds, fous, pauvres, et autres déviances. D'où venaient ces populations? N'existaient-elles pas avant le $\mathrm{XIX}^{\mathrm{e}}$ siècle sous une forme ou une autre ? Selon Hacking, les choses que les gens faisaient pour atterrir dans telle ou telle catégorie existaient auparavant (voler, tuer, boire, etc.) mais l'utilisation de ces choses pour décrire, énumérer et classifier les gens est artificielle et change souvent. Il soutient que : 
[...] même les recensements provinciaux ou nationaux montrent de façon extraordinaire que les catégories sous lesquelles tombent les populations changent tous les dix ans. Les changements sociaux créent de nouvelles catégories de population, mais le décompte est loin d'être un simple compte-rendu des évolutions. Ils créent de façon élaborée et souvent philanthropique de nouveaux modes d'être de la population. (Hacking, 2002 : 100)

Les catégories que Hacking évoque impliquent différentes " façons d'être » telles que la sexualité, l'ethnie ou la race. Hacking, par exemple, écrit que "l'homosexuel et I'hétérosexuel en tant que types de personnes (façons d'être une personne, ou conditions d'identité individuelle) ne sont apparus qu'à la fin du XIX ${ }^{e}$ siècle » (Hacking, 2002 : 103). Auparavant, il existait des actes sexuels entre individus mais ceux-ci ne sont tombés sous le coup de descriptions familières et reconnaissables (dicibles et visibles) qu'à partir du $\mathrm{XIX}$ siècle. Et il $n^{\prime}$ existe aucune garantie que ces descriptions resteront telles quelles $a d$ vitam eternam. Son argument " [...] n'est pas qu'il existe un type de population qui s'est vu de plus en plus reconnu par les bureaucrates ou les étudiants de la nature humaine, mais plutôt qu'un type de personne est advenu en même temps que le type lui-même a été inventé » (Hacking, 2002 : 106).

Ainsi, l'argument n'est pas qu'un type de peuple n'a jamais existé auparavant et est advenu à un certain moment. Ni qu'un type de peuple a toujours existé et qu'il a été nommé à un moment précis. Mais plutôt qu'à un certain moment de l'histoire, les peuples ne se voyaient pas de cette façon particulière, $n$ 'interagissaient pas de cette façon avec les autres peuples, et n'étaient pas traités par la connaissance, les institutions, les autorités en fonction de cette description. Ainsi, façonner un peuple est un événement performatif complexe qui nécessite une enquête d'ontologie historique.

Lorsque Hacking parle de " façonner les gens ", il parle de types de peuples. II existe des types de peuples tels les assassins, dandys, badauds, Noirs, homosexuels, et ainsi de suite qui constituent " partie » d'un " tout». Ces types de peuples en tant que " partie » sont inconcevables sans les types de peuple qui se sont constitués en "tout». Voilà un problème que Hacking ne traite pas. Un peuple (ou des peuples) peut être décrit seulement lorsqu'il existe une différence entre les peuples (les parties) et le peuple (le tout). Le peuple en tant que tout suppose une ontologie historique plus complexe que Hacking ne l'entend, notamment la relation entre tout et parties. La raison en est que les interactions entre les mots et les choses selon Hacking impliquent des rapports de force, et qu'il existe des jeux de domination à l'intérieur de ces descriptions. La description « noir » ou " gay » n'est pas seulement une description en fonction de laquelle un peuple agira mais également un signifiant asymétrique en fonction duquel on agira sur ces peuples - qui seront sujets au gouvernement. Par ailleurs, c'est une chose de voir comment la catégorie des assassins s'est créée, c'en est une autre de comprendre comment la catégorie juif ou français est advenue. La question qui en découle n'est pas seulement l'invention de types de peuples mais également l'invention du peuple. En d'autres termes, la question "qu'est-ce qu'un peuple? " mène inévitablement à la question " qu'est-ce que le peuple? ". 
Hannah Arendt dans les années 1940 et Michel Foucault dans les années 1970 sont arrivés à ce problème de la différence entre un peuple et le peuple (Isin, 2012). Arendt est connue pour avoir soutenu que c'est au cours du XIX ${ }^{e}$ siècle que la nation en tant que peuple a conquis l'État. Sa généalogie de la pensée raciale, par exemple, l'a amenée à comprendre les dangers de l'institution d'un État en tant que nation. De même, Foucault se pencha sur la façon dont les nations deviennent la nation au XIX ${ }^{e}$ siècle. Pour les deux penseurs, façonner les peuples nations en Europe a généré des peuples « minorités ». Ce que nous avons appris de Arendt et Foucault est qu'une généalogie " du peuple » mène inéluctablement aux types de peuples qu'elle génère.

\section{Qu'est-ce que le peuple?}

Comment un peuple devient-il un peuple ? Un peuple devient-il uniquement le peuple qu'il est ? Si c'est le cas, alors qu'est-il ? Comment pouvons-nous savoir : 1) ce qu'est un peuple en général, 2) ce qu'est ce peuple-ci ou ce peuple-là, 3) ce que nous-mêmes sommes? (Heidegger, 2012)

II n'est peut-être pas surprenant qu'avant Arendt dans les années 1940 et Foucault dans les années 1970, Martin Heidegger ait été confronté dans les années 1930 à la question de la différence entre un peuple et le peuple. Pour illustrer les difficultés d'appréhender le concept de "peuples mobiles", nous aurions besoin d'une ontologie historique du demos des Grecs de l'Antiquité, de la plèbe chez les Romains et du popolo italien du Moyen Âge pour nous montrer comment ces différents peuples sont devenus des reliquats ou résidus du peuple. Margaret Canovan (2005) retrace justement avec précision cette histoire. Elle met au jour une tension entre "le peuple», qui se différencie des populations turbulentes, pauvres, exaltées et impétueuses. Elle note que les histoires de respectabilité, d'union et de vertu «du peuple» vont toujours à l'encontre d'une populace ou horde déchaînée. Canovan dispense déjà l'idée que la différence entre un peuple et le peuple, voire le passage d'un peuple au peuple, présuppose la domination. Alain Badiou ne dit pas autre chose :

Le mot " peuple » ne convenait qu'aux puissants conquérants, exaltés par la conquête même: "peuple français », " peuple anglais », oui... Peuple algérien ou vietnamien, non ! Et pour le gouvernement israélien, encore aujourd'hui, " peuple palestinien », pas davantage ! (Badiou, $2016: 22-23$ )

C'est pourtant Giorgio Agamben (2000) qui a identifié cette tension comme question constitutive des politiques « occidentales ». Il souligne :

[...] toute interprétation du sens politique du mot « peuple » doit partir du fait singulier que, dans les langues européennes modernes, il désigne toujours également les pauvres, les déshérités, les exclus. Un même mot recouvre aussi bien le sujet politique constitutif que la classe qui, de fait sinon de droit, est exclue de la politique. (Agamben, $2000: 29)$

Dès que commence la réflexion politique sur le "peuple ", apparaît une ambiguïté sur le concept, à savoir s'il recouvre le dominant constitutif (le peuple), le dominé (une population) ou les deux. D’après Agamben : 
Une ambiguïté sémantique aussi répandue et constante ne peut être fortuite : elle doit refléter une amphibologie inhérente à la nature et à la fonction du concept de peuple dans la politique occidentale. (Agamben, $2000: 31$ )

Toujours selon lui :

Cela signifie aussi que la constitution de l'espèce humaine en un corps politique passe à travers une scission fondamentale et que dans le concept "peuple " nous pouvons reconnaître sans difficulté les couples catégoriels qui définissent la structure politique originelle : vie nue (peuple) et existence politique (Peuple), exclusion et inclusion, corps naturel et corps politique. (Agamben, $2000: 31-32$ )

Il en conclut :

En fait, le « peuple » porte depuis toujours en lui la fracture biopolitique fondamentale. Il est ce qui ne peut être inclus dans le tout dont il fait partie et il ne peut appartenir à l'ensemble dans lequel il est déjà inclus. (Agamben, 2000 : 32, en italique dans le texte)

Ainsi, "s'il est vrai que le peuple contient nécessairement en lui-même la fracture biopolitique fondamentale, il sera alors possible de lire d'une nouvelle manière quelques pages décisives de l'histoire de notre siècle "(Agamben, 2000 : 33). Alors même que Agamben identifie cette fracture et souligne la nécessité de lire d'une nouvelle manière certains événements $d u X X{ }^{e}$ siècle, il sous-entend également que cette fracture est si fondamentale qu'elle nécessite le réexamen de l'histoire politique " occidentale " toute entière. Je suis conscient du prodigieux débat que suscitent les interprétations de Agamben sur Arendt et Foucault et de sa différenciation entre zoē, le corps naturel, et bios, le corps politique, mais je considère son argument sur la fracture fondamentale dans la théorie politique occidentale comme un défi réel de la réflexion généalogique sur un peuple.

Je placerai les peuples mobiles au sein de cette fracture après avoir évoqué Jacques Rancière et Ernesto Laclau, qui ont sérieusement tenu compte de ce défi. Ils partent tous deux de l'hypothèse qu'il existe de fait une fracture ou tension fondamentale entre " le peuple » en tant que tout et un peuple ou des peuples en tant que ses parties. J'aborderai brièvement la façon dont chacune de ces tentatives cherche à travailler cette tension pour l'utiliser de façon critique dans une réflexion sur le "peuple » en tant que concept politique.

La description de Rancière du tout et des parties dans la politique de l'Antiquité est bien connue. Je résumerai brièvement ci-après son traitement de la fracture fondamentale de la politique occidentale identifiée par Agamben. Si en effet il est possible de décrire le " tout " comme une police et des " parts " comme ses éléments constitutifs, comment leur relation se décide-t-elle ? Chez Rancière, la politique naît du litige portant sur le compte de ses parties. Selon lui, les interprétations dominantes de la politique antique comprenaient jusqu'alors cette politique comme émanant d'une police déjà constituée et de ses conflits préexistants; cependant, poursuit-il, c'est l'inverse qui est vrai. Toute police se fonde sur la politique émanant de ce qu'il appelle le compte des parts la constituant. Il y a de la politique quand sont comptés les parts ainsi que ses litiges sur le compte des parts constituant la police. 
Rappelons-nous que, chez Rancière, il existe deux façons de compter : arithmétique et géométrique. Ces deux méthodes ne sont pas tant des descriptions du compte que des démarches qui permettent le compte. Ainsi, Rancière peut schématiser deux façons de penser la politique : un compte arithmétique présumant que tout est comptabilisé (d'où un mécompte) et un compte géométrique qui compte les sans-part. Quand le compte est arithmétique (soit ce qui est compté), il rend compte de ce qui est donné ; quand le compte est géométrique, il rend compte de ce qui n'est pas donné. Cependant, et c'est le point crucial, les comptes sont toujours des mécomptes puisqu'ils ne prennent pas en considération ce qui compte réellement. La politique provient de ce paradoxe d'incapacité à et de nécessité de compter les parts (Rancière, $1998: 6$ ). II y a de la politique quand les sans-part luttent pour se faire compter (Rancière, 1998 : 9). Se faire compter est un acte politique car ces parts ne disposent pas de part à laquelle s'identifier pour être le tout policier. C'est cette identification audacieuse qui est politique. Elle prend, par exemple, la signification historique du demos dans la politique athénienne. La revendication du demos n'est pas seulement de se faire compter mais également de se constituer comme le tout. $C^{\prime}$ est ainsi que la langue des sans-part n'est pas une lutte essentielle entre les riches et les pauvres, entre telle ou telle classe, ou entre tel ou tel groupe social. La politique n'est pas une opposition entre riches et pauvres. Au contraire, la politique est l'interruption d'un ordre de domination par l'institution de la part des sans-part (Rancière, 1998 : 11).

Pour l'instant, dans ce résumé, nous avons parlé des peuples et du peuple. Pour traduire la langue du tout et des parties en catégories de peuples et du peuple, Rancière introduit deux termes : égalité et tort. La lutte des sans-part à s'instituer en tant que partie se base sur une demande d'égalité par suite de la déclaration d'un tort, d'une injustice. La demande d'égalité est nécessaire à la déclaration du tort et cette déclaration est impossible sans l'hypothèse de l'égalité de parole et de capacité, à parts égales dans le litige. Si en effet « la politique existe là où le compte des parts et des parties de la société est dérangé par l'inscription d'une part des sans-part. Elle commence quand l'égalité de n'importe qui avec n'importe qui s'inscrit en liberté du peuple " (Rancière, 1998 : 123). Et " cette liberté du peuple est une propriété vide, une propriété impropre par quoi ceux qui ne sont rien posent leur collectif comme identique au tout de la communauté " (Rancière, 1998 : 123-124). Ainsi, le passage d'un peuple au peuple, sa revendication, est à l'origine de la politique (ou comment la politique « occidentale » perçoit ses origines).

Quel est ce tout auquel un peuple s'identifie ? Rancière écrit que " le peuple " a une double incarnation : c'est à la fois le nom de la police tout entière et le nom d'une partie de cette police. L'écart entre ces deux dénominations du peuple est le lieu d'une doléance (Rancière 1995 : 97). De notre perspective de pensée sur les peuples mobiles comme un peuple, l'argument principal de Rancière est le suivant. Si la politique antique comprenait cet écart, la politique moderne ne peut le tolérer. La politique moderne ne peut accepter que le peuple soit à la fois et en même temps dominant et dominé, tout et partie, sédentaire et mobile (Rancière, 1995 : 97). Pour Rancière, dans la politique moderne, "l'apparence du peuple doit s'en tenir strictement aux attributs de la souveraineté ou à l'apparence de la souveraineté dissoute en faveur des réalités du peuple producteur » (Rancière, 1995 : 99). Ainsi, cet écart entre un peuple et le peuple est à la fois un défi et un 
déclencheur de politique; les anciens l'avaient compris, les modernes ne peuvent le tolérer.

Laclau, sans pour autant s'intéresser aux origines antiques ou lignages de la politique moderne, est très proche de Rancière dans sa tentative d'utiliser cette tension pour comprendre ce qui est fondamental dans la politique. Au centre de son argumentation est le concept sociopolitique de "demandes » articulé par le dominé envers l'ordre dominant (hégémonique). Le dominé articule une exclusion ou privation de sa doléance et cette articulation comme demande constitue un peuple (Laclau, 2005 : 123). Comme chez Rancière, un peuple n'est donc pas un concept sociologique préexistant mais quelque chose qui provient de l'intérieur de la politique. Ce raisonnement tient compte d'une asymétrie constitutive entre la police entendue comme tout (le populus) et les dominés comme parties de ce tout (la plèbe). Pour Laclau comme pour Rivière, il est crucial que la plèbe s'identifie au populus comme police d'un tout (Laclau, 2005 : 224). Ainsi, comme chez Rancière, la plèbe fonctionne comme partie d'un tout et en même temps comme partie qui est le tout (Laclau, 2005 : 225). La logique de l'hégémonie, découlant de cette tension entre la partie et le tout, suppose que le tout est " contaminé " par la partie et que la partie comprend le tout. La distinction analytique entre l'universel et le particulier comme contraires mutuellement exclusifs est ainsi fausse et dément la logique de l'hégémonie (Laclau, 2005 : 226). Pour Laclau, l'ambiguïté " du peuple » à la fois comme populus et plèbe n'est pas une contradiction logique mais exprime la logique de I'hégémonie. Là où Laclau diffère de Rancière, c'est quand Rancière semble présupposer que la constitution des sans-part en appellera toujours à une politique d'émancipation, alors que Laclau conteste que ce point puisse être déterminé théoriquement (Laclau, 2005 : 246). À la différence de Rancière, Laclau insiste aussi sur les limites de l'analyse philosophique et sur la nécessité d'enquêtes sociologiques sur les façons dont la logique de l'hégémonie constitue un peuple (Laclau, 2005 : 248).

Ce qui est important à la fois chez Rancière et Laclau, de mon point de vue, c'est leur insistance à considérer la tension entre les parties et le tout comme tension constitutive de la politique. Cependant, il m'est difficile d'accepter cette insistance selon laquelle la politique implique inéluctablement, sinon intrinsèquement, la construction ou formation d'un peuple comme un tout, c'est-à-dire le peuple. Plus spécifiquement, le préalable de l'identification des parties au tout pour y prendre part justifie toujours l'advenir d'un type de peuple que le peuple est d'ores et déjà. $\mathrm{Si}$, comme l'affirme Rancière, il n'y a pas de politique au-delà et en dehors de cette configuration du tout et de ses parties et qu' « il n'y a que l'ordre de la domination et le désordre de la révolte ", comment alors la configuration s'interrompt-elle (Rancière, 1998 : 12) ? Si nous soutenons avec Rancière et Laclau que le tout (le peuple) et ses parties (peuples) sont imbriqués l'un dans l'autre, peut-on alors imaginer un peuple qui ne s'identifie pas au peuple ? Tous deux, Rancière et Laclau, définissent la politique comme une rupture dans un ordre donné mais n'expliquent jamais pourquoi cette rupture suppose nécessairement la formation du peuple, l'identification au tout. Selon Laclau, la formation d'un peuple implique un acte d'institution et, en tant qu'acte, qu'il ne tire pas sa force " de toute logique fonctionnant déjà dans la situation précédente " et que "ce qui est crucial pour l'émergence "du 
peuple", en tant que nouvel acteur historique, est que l'unification de la pluralité des demandes dans une nouvelle configuration est constitutive et non dérivative " (Laclau, 2005 : 228). II semblerait que Laclau surmonte cette tension, et nous pouvons en apportant quelques modifications nous accorder avec lui. Mais il ajoute ensuite que " cela constitue un acte dans le sens strict du terme, puisqu'il ne prend pas sa source dans une chose externe à lui-même " (Laclau, 2005 : 224). Que nous travaillions cette tension entre un peuple (ou peuples) et le peuple comme écart constitutif de la politique (Rancière) ou comme contamination constitutive de la police (Laclau), nous restons face à une fracture fondamentale qui ne prend son sens que contre un tout déjà constitué. II ne reste dès lors aux peuples mobiles qu'une alternative, devenir sédentaires ou rester en dehors de la politique.

\section{Peuples mobiles : configurations transversales}

Deux obstacles se dressent si l'on tente de comprendre les peuples mobiles avec Rancière et Laclau, et, au demeurant, avec Agamben voire Hacking. D'abord, et ce dans tous leurs travaux de théorisation, un peuple demeure sans géographie. Chacun met l'accent sur l'importance de comprendre un peuple avec son histoire mais certains peuples restent sans géographie. Mettant en garde contre l'écriture de peuples sans histoire, dans la pratique chacun traite les peuples sans géographie. Ce que j'entends par là peut être illustré par la remarque de James Scott: "Un grand nombre de noms apparemment ethniques s'avèrent, une fois traduits, être une description de la géographie d'un peuple, appliqués à eux par le discours de l'État : "peuple des collines", "habitants des marais", "peuple des bois", "peuple des steppes" „(Scott, 2017). Où nous pouvons ajouter d'autres exemples contemporains: peuple sans adresse permanente, peuple sans-papiers, peuple sans propriété, peuple sans nationalité, et, le plus troublant d'entre eux, peuple sans État. Gilles Deleuze et Félix Guattari (1987) déclaraient qu' « on écrit l'histoire, mais on l'a toujours écrite du point de vue des sédentaires, et au nom d'un appareil unitaire d'État, au moins possible même quand on parlait des nomades ". Nous avons besoin de voir que la géographie - les façons dont les peuples se constituent dans l'espace - est tout aussi constitutive que l'histoire à l'advenir d'un peuple. Ce qui singularise les peuples comme peuples des collines, des bois ou sans-papiers, c'est qu'ils sont décrits par des peuples sédentaires, c'est-à-dire du point de vue des États organisés en tant que territoires limités. En dépit de toutes les tensions, contaminations, fractures et écarts identifiés entre le tout et ses parties par Agamben, Rancière et Laclau, ce qui n'est pas reconnu, c'est que, du point de vue d'un État, un « vrai » peuple correspond toujours à un territoire donné. La généalogie du concept de territoire de Stuart Elden (2013) documente minutieusement cette production sociale. II retrace la façon dont le discours juridico-politique produit un État en tant que territoire délimité et comme nom de cet espace. II nous faut admettre que la pensée politique "occidentale " tient pour évident ce concept de territoire, le considérant même comme un État, conformément à la description de Scott (1999). Mais si l'on prend une autre position (voir ci-dessous), les sans-part sont souvent des peuples mobiles domestiqués pour devenir partie du peuple. Les résidus du tout, les sans-part restent des peuples mobiles : nomades, marins, pirates, 
voyageurs, migrants, réfugiés, itinérants, tziganes, vagabonds. Ces peuples mobiles sont dans l'impossibilité de se constituer comme sujets politiques précisément car ils ne peuvent correspondre à un territoire, puisqu'ils restent des peuples sans géographie. L'idée du territoire, comme espace délimité sous le contrôle d'un peuple organisé par le truchement d'un État qui les constitue comme le peuple de cet État, reste l'image dominante de la pensée politique; or dépasser cette image (par exemple en pensant différemment les histoires et géographies des peuples) est une tâche ardue.

Le deuxième obstacle à la compréhension de la fracture entre les peuples et le peuple est que la différence se situe rarement entre un peuple qui se constitue en dominants (le peuple) et l'autre en dominés (des peuples). La réalité est souvent plus subtile que ce que laisse entendre cette opposition. II s'agit plutôt d'un spectre de différents peuples et de la façon dont les individus prennent position (et en donnent) sur ce spectre : inconnus, de l'extérieur, étrangers. Souvent, les peuples passent (ou se retrouvent dans) de multiples positions qui se recoupent sur ce spectre (Isin, 2002, 2017). Comme Scott (2017) l'écrit, « il est donc clair que nombre de barbares n'étaient pas des primitifs arriérés ou marginalisés, mais plutôt des réfugiés politiques et économiques ayant fui vers la périphérie afin d'échapper à la pauvreté, à l'impôt, à la servitude et aux guerres liés à l'existence de l'État ${ }^{4}$ ». Les citoyens peuvent devenir des barbares et les barbares peuvent devenir des citoyens.

Le travail de Scott sur l'histoire des premiers États apporte une contribution majeure à la réflexion sur l'advenir d'un peuple, et sur la différenciation entre le peuple et des peuples souvent établie par antinomie: nomade/sédentaire, État/non-État, civilisé/barbare (Scott, 2009, 2017). Scott (2017) reconnaît que l'essence de ce que nous savons de la géographie historique a été écrite du point de vue des peuples sédentaires installés dans chaque territoire État. La prise de contrôle d'un territoire par ces peuples, tissant des patchworks d'arrière-pays et périphéries et assujettissant les peuples de ces espaces pour les faire devenir peuples d'État, nous montre que les peuples assujettis étaient invariablement des peuples mobiles, barbares, qui n'étaient pas simplement en dehors de l'État mais qui furent assujettis en catégories différenciées. Scott utilise le mot "barbare" comme concept générique de peuples mobiles que les États étaient incapables d'assujettir, ou, et c'est encore plus remarquable, comme peuples résiduels créés par les États eux-mêmes et qui échappaient à l'assujettissement. Le récit apparemment simple mais efficace de Scott raconte comment nous pourrions voir I'histoire du point de vue des assujettis, géographie historique des peuples mobiles. Scott martèle que l'histoire a été écrite du point de vue des peuples sédentaires qui représentent la norme. Cependant, comme il l'illustre, depuis l'émergence des premiers États (pas seulement entre le Tigre et l'Euphrate mais aussi sur les bords du fleuve Jaune et dans les plaines alluviales de l'Amérique du Sud au cours des 10000 dernières années), c'est seulement au cours des trois ou quatre derniers siècles que les États et leurs peuples sédentaires se sont établis comme norme. Or, en ce qui concerne l'histoire " profonde "

${ }^{4}$ Trad. Marc Saint-Upéry, Éd. La Découverte, 2019. 
des États, il s'agit d'un fait relativement récent et exceptionnel. II convient toutefois de faire preuve de retenue en reconnaissant les peuples sédentaires comme la norme des 400 dernières années. La prolifération des migrants et réfugiés, particulièrement au cours des deux derniers siècles, et les déplacements et bouleversements violents de millions de personnes devraient nous inciter à réfléchir à deux fois avant d'accepter les États de peuples sédentaires comme norme (Jones, 2016 ; Mazard, 2014). En outre, Scott dépeint une différence trop criante entre les barbares comme peuples non étatiques par opposition aux peuples étatiques. Cependant, comme il l'illustre, les barbares comprennent différents peuples dont les relations aux États sont toujours en flux. De tels peuples, comme je l'ai affirmé ci-dessus, sont entrés et sortis des positions de barbares : nomades, sauvages, pirates et autres. C'est une des raisons pour lesquelles je préfère envisager les peuples mobiles en tant que "configurations transversales" dont les affiliations, appartenances, affinités et mouvements traversent et sont à l'intersection entre différentes frontières et limites, établissent des associations, assemblages et solidarités grâce auxquels ils agissent en tant que sujets politiques. Malgré tout, la critique de Scott de ce grand récit des États aux peuples sédentaires nous apporte une ontologie historique des peuples mobiles de longue durée $e^{5}$ qui brosse un tableau bien différent.

Si les peuples mobiles doivent devenir norme et non plus exception, sujets politiques et non plus peuples sujets, il nous faut commencer par une image de la pensée observant le processus de constitution des peuples mobiles en sujets politiques, non pas dans le cadre de territoires délimités mais par des configurations transversales. Comme je l'ai dit tantôt, la question de savoir comment les peuples mobiles sont devenus une exception doit être traitée avant toute chose. Comment les peuples sédentaires ont-ils pu devenir la norme qui constitue les peuples mobiles en exception ? Or, ceci nous amène à nous demander pourquoi nous constituons à présent les peuples mobiles comme norme. Pour peu, en effet, qu'il nous soit impossible de recourir à des faits indépendants sur les peuples mobiles comme nouvelle norme, puisqu'un tel recours reflète notre manière de collecter, compiler, assembler et interpréter ces faits. Ainsi, il nous est impossible de nous placer en dehors du processus dynamique décrit par Hacking sur l'advenir des types de peuples: prémices d'une description, peuple agissant selon cette description, formation d'institutions pouvant prendre en charge les peuples agissant de la sorte, accumulation de connaissance sur le peuple agissant de la sorte, et réglementation du peuple par l'expertise, l'autorité, le pouvoir. Si nous façonnons des peuples mobiles comme norme plutôt qu'exception, nous rentrons dans cette dynamique par laquelle les peuples mobiles deviennent sujets politiques de et assujetis à de nouveaux modes de gouvernement.

\section{Remerciements}

Cet essai s'appuie sur une proposition du projet Leverhulme Doctoral Scholarship élaborée par la Queens Mary University of London. Je remercie tous mes collègues dans

\footnotetext{
${ }^{5}$ En français dans le texte.
} 
les différentes écoles et les différents départements qui ont commenté les versions précédentes de la proposition soumise au Leverhulme Trust. J'aimerais tout particulièrement remercier Kim Hutchings et Jef Huysmans pour les points pertinents qui ont permis le développement du projet. Ma reconnaissance va également à mes collègues de la School of Politics and International Relations (SPIR) pour leurs commentaires d'une conférence basée sur cet article. Mes remerciements vont également au Leverhulme Trust pour $\mathrm{m}$ 'avoir permis de travailler sur ce projet. J'aimerais remercier les trois réviseurs anonymes pour leurs commentaires et critiques et les éditeurs du numéro thématique pour leurs conseils. Enfin, je tiens à remercier Eugene Brennan pour avoir minutieusement vérifié la traduction française et les traducteurs pour leur excellente traduction.

\section{Bibliographie}

Adey P., Bissell D., Hannam K., Merriman P., Sheller M. (Eds.), 2014, The Routledge handbook of mobilities, London, Routledge.

Agamben G., 2000, Means without end: Notes on politics, Minnesota, University of Minnesota Press.

Austin J.L., 1962, How to do things with words, Oxford, Oxford University Press.

Badiou A., 2016, "Twenty-four notes on the uses of the word "people" ", in B. Bosteels, K. Olson (Eds.), What is a people?, New York, Columbia University Press, pp. 21-31.

Boswell C., 2009, The political uses of expert knowledge: Immigration policy and social research, Cambridge, Cambridge University Press.

Buscher M., Urry J., Witchger K. (Eds.), 2011, Mobile methods, London, Routledge.

Canovan M., 2005, The people, Cambridge, Polity.

Cresswell T., 2006, On the move: Mobility in the modern Western world, London, Routledge.

Deleuze G., Guattari F., 1987. A thousand plateaus: Capitalism and schizophrenia, Minneapolis, University of Minnesota Press.

Elden S., 2013, The birth of territory, Chicago, The University of Chicago Press.

Elliott A., Urry J., 2010, Mobile lives, London, Routledge.

Endres M., Manderscheid K., Mincke C. (Eds.), (2016, The mobilities paradigm: Discourses and ideologies, London, Routledge.

Feldman G., 2015, We are all migrants, Stanford, CA, Stanford University Press.

Foucault M., 1997, "What is enlightenment? ", in Ethics: Subjectivity and truth (the essential works of Michel Foucault, 1954-1984), vol. 3, New York, New Press, pp. 303-320.

Frello B., 2008, "Towards a discursive analytics of movement: On the making and unmaking of movement as an object of knowledge ", Mobilities, $n^{\circ} 3$, pp. 25-50.

Gabaccia D.R., 1999, "Is everywhere nowhere? Nomads, nations, and the immigrant paradigm of United States history ", The Journal of American History, $n^{\circ}$ 86(3), pp. 1115-1134. 
Geiger M., 2013, "The transformation of migration politics: From migration control to disciplining mobility ", in M. Geiger, A. Pécoud (Eds.), Disciplining the transnational mobility of people, Basingstoke, Palgrave Macmillan, pp. 15-40.

Gold S.J., Nawyn S.J. (Eds.), 2013, Routledge international handbook of migration studies, London, Routledge.

Hacking I., 2002, Historical ontology, Cambridge, Harvard University Press.

Hacking I., 2007, "Kinds of people: Moving targets ", Proceedings of the British academy, $n^{\circ} 151$, pp. 285-318.

Hannam K., Mostafanezhad M., Rickly-Boyd J.M. (Eds.), 2016, Event mobilities: Politics, place and performance, London, Routledge.

Heidegger M., 2012, Contributions to philosophy (of the event) 1936-38, Indianapolis, Indiana University Press.

Isin E.F., 2002, Being political: Genealogies of citizenShip, Minneapolis, University of Minnesota Press.

Isin E.F., 2012, "Citizens without nations ", Environment and Planning D: Society and Space, $\mathrm{n}^{\circ} 30(1)$, pp. 450-467.

Isin E.F., 2017, "Performative citizenship », in A. Shachar, R. Bauböck, I. Bloemraad, M. Vink (Eds.), The Oxford handbook of citizenship, Oxford, Oxford University Press, pp. 500-523.

Isin E.F., Nyers P. (Eds.), 2014, Routledge handbook of global citizenship studies, London, Routledge.

Jones R., 2016, Violent borders: Refugees and the right to move, London, Verso.

Laclau E., 2005, On populist reason, London, Verso.

Lucassen J., Lucassen L., 2017, "Migration over cultural boundaries: A rejoinder ", International Review of Social History, $n^{\circ}$ 62(3), pp. 521-535.

Lucassen J., Lucassen L. (Eds.), 2014, Globalising migration history: The Eurasian experience (16th21st centuries), London, Routledge.

Manderscheid K., 2016, "Who does the move? Affirmation or deconstruction of the solitary mobile subject ", in M. Endres, K. Manderscheid, C. Mincke (Eds.), The mobilities paradigm: Discourses and ideologies, London: Routledge, pp. 91-113.

Mazard M., 2014, "The art of (not) looking back: Reconsidering Lisu migrations and "Zomia" ", in J. Lucassen L. Lucassen (Eds.), Globalising migration history: The Eurasian experience (16th-21st centuries), London, Routledge, pp. 215-246.

Merriman P., 2012, Mobility, space, and culture, New York, Routledge.

Nietzsche F.W., 2001, The gay science: With a prelude in German rhymes and an appendix of songs, Cambridge, Cambridge University Press.

Osterhammel J., 2009, The transformation of the world: A global history of the nineteenth century, Princeton, Princeton University Press.

Panayi P., Virdee P. (Eds.), 2011, Refugees and the end of empire: Imperial collapse and forced migration in the twentieth century, Basingstoke, Palgrave Macmillan. 
Pécoud A., 2015, Depoliticising migration: Global governance and international migration narratives, Basingstoke, Palgrave Macmillan.

Rancière J., 1995, On the shores of politics, London, Verso.

Rancière J., 1998, Disagreement: Politics and philosophy, Minneapolis, University of Minnesota Press.

Scott J.C., 1999, Seeing like a state: How certain schemes to improve the human condition have failed, New Haven, Yale University Press.

Scott J.C., 2009, The art of not being governed: An anarchist history of upland Southeast Asia, New Haven, Yale University Press.

Scott J.C., 2017, Against the grain: A deep history of the earliest states, New Haven, Yale University Press.

Organisation des Nations unies (ONU), 2016, « 244 million international migrants living abroad worldwide, new UN statistics reveal », 12 January, http://wp.me/p5Mdaw-3EG (consulté le 03/11/2019).

Zanoni E., 2017, "Thinking big about mobile people ", Journal of American Ethnic History, ${ }^{\circ}$ 37(1), pp. 57-64. 